\title{
El sistema antártico y la cuestión del aprovechamiento de los recursos en el área
}

\section{INTRODUCGION}

1. La iniciativa del "Law Review" de la Universidad de Miami de tratar en un número especial los problemas actuales de la Antártica, es digna de encomio y puede contribuir a esclarecer un tema que es abordado a menudo con insuficientes antecedentes o cierta ligereza de juicio.

El autor de estas líneas ha aceptado con agradecimiento la invi-. tación que se le hizo para que tormulase algunos comentarios sobre este importante tema. Lillos serán de naturaleza muy general, dado el apremio del tiempo, y tendrán carácter estrictamente personal, sin reflejar necesariamente la posición de Chile, país al que ha representado en varias reuniones del Tratado Antártico.

2. El interés por la Antártica ha aumentado considerablemente en los últimos años, en la medida en que se ha pasado a estimar como más posible, o más rentable, un aprovechamiento de sus recursos.

La creación de un nuevo orden jurídico para los océanos, y especialmente la universalización de la Zona Económica Exclusiva. de 200 millas, ha originado el desplazamiento de las grandes tiotas pesqueras de agua distante hacia nuevas áreas del planeta. En esta perspectiva, las aguas antárticas - $y$, sobre todo, las concentraciones del pequeño crustáceo llamado "krill"-, han cobrado un creciente interés.

La llamada "crisis del petróleo" y sus consecuencias, por otra parte, motivó un nuevo examen de las potencialidades de hidrocarburos en la región antártica y, más concretamente, en sus plataformas continentales.

Aun cuando las dimensiones de la riqueza pesquera de la zona, y concretamente las del krill, sean bastante desconocidas y muchisimo más modestas que las estimaciones formuladas en reportajes un tanto sensacionalistas; y aun cuando la explotación mineral en la Antártica es una posibilidad tecnológicamente difícil y económicamente imposible por ahora, es un hecho que periodistas, políticos, hombres de negocios y representantes del pensamiento han 
acentuado su concentración en esta área, habitualmente muy olvidada en los últimos años.

3. Esta toma de conciencia acerca de las potencialidades econón. micas de la Antártica, ha relegado a un segundo plano otras consideraciones que primaron a su respecto en el pasado y que nunca debieran estar ausentes de quienes efectivamente se preocupan del bienestar de la Humanidad.

La Antártica fue la primera zona de paz y la primera área desnuclearizada que existió en el mundo, superando las potencialidades bélicas que se insinuaron primero con ocasión de la Segundá Guerra Mundial y luego cuando se agudizó la llamada "guerra fría". El régimen allí establecido sirvió de precedente a la creación de otras zonas desnuclearizadas y de paz, tales como la originada por el Tratado de Tlatelolco para la América Liatina.

La zona antártica ha sido la primera - y tal vez la única- reserva ecológica que se ha dado la Humanidad, cuya creactón fue muy anterior a la creciente preocupación universal por la defensa y preservación del medio ambiente.

Fue y es Lambién un laboratorio científico, abierto a todos los hombres. Los resultados de la enorme investigación llevada a cabo en la Antártica - no sólo respecto a sus propias características sino a la relación de éstas con el clima y otros problemas universales-, han sido puestos a disposición general y enriquecido así el acervo đe conocimiento del mundo.

Estas y otras realidades del Continente helado han pasado recientemente a un segundo plano y se encuentran, por desgracia, un poco olvidadas.

4. Se ha comparado a la Antártica con otras así llamadas "Fronteras de la Humanidad": el espacio exterior o ultraterrestre y los fondos marinos y oceánicos situados fuera de la jurisdicción nacional1.

Más allá del interés intelectual que esta analogía suscita y aun reconociendo ciertos elementos que los tres espacios mencionados puedan tener en común, es clarísimo que existen diferencias de enorme magnitud, que superan cumplidamente a las posibles semejanzas.

Es preciso comprobar, en primer lugar, que el espacio exterior

IJessup and Taubenfeld. "Controls for Outer Space and the Antarctic Analogy" - New York 1959 - Columbia University Press.

"The Role of the Developing Nations in the Formulation of International Controls for Unoccupied Regions: Outer Space, the Ocean Floor, and Antarctica", by Richard Newton Bing - Tufts University - June 1972. 
y los fondos marinos extrajurisdiccionales no han sido ni deberian ser objeto de ocupación exclusiva o soberanía; puede estimárseles, según se ha declarado y propiciado en las Naciones Unidas, como "patrimonio común de la Humanidad", en tanto que para algunos continuarán siendo "res nullius". Esto no ocurre, de ninguna ma= nera, en la Antártica, en la cual existen afirmaciones de soberanía de carácter secular y en donde siete Estados la ejercen o reclaman en la actualidad.

En segundo término, cabe llegar a Ia conclusión de que, a lo. menos por ahora, no hay propiamente un régimen jurídico para. el espacio ultraterrestre o los fondos marinos más allá de la jurisdicción nacional, si bien se han proclamado principios que constituyen un embrión de dichos regímenes para ambas situaciones. En la Antártica, en cambio, existe un régimen jurídico establecido por el llamado "sistema antártico" —es decir, el Tratado Antártico y su desarrollo en las Reuniones Consultivas del mismo-, aun cuando éste sea incompleto.

En la Antártica - por último- hay actividades del hombre desde muy antiguo, que han adquirido gran intensidad en este siglo, en los campos de la exploración, el conocimiento geográfico, la investigación científica, el turismo, la meteorología y aún el aprovechamiento de los recursos (focas, ballenas). En los casos del espacio ultraterrestre y los fondos marinos extrajurisdiccionales, no. existe, prácticamente, dicha actividad.

La Antártica no es, no puede estimársele, como "res nullius" ni como una zona situada más allá de la actividad y la reglamentación jurídica del hombre. Existe en ella un régimen jurídico con un avanzado grado de madurez y vigencia; se aplica a ese Conti-. nente soberanía o, a lo menos, manifestaciones de la misma.

5. Interesa a toda la Humanidad que pueda preservarse la obra admirable realizada en la Antártica, que será tratada más adelante.

Es necesario, antes que nada, que el aprovechamiento de los recursos se haga sin afectar sustancialmente la ecología antártica, las ecologías vecinas, que están directamente relacionadas con ella, y: la del mundo en general. El Gontinente antártico es posiblemente la última área de nuestra Naturaleza preservada como tal, en su primitivo equilibrio, sin elementos perturbadores o destructores.

También es de la mayor importancia preservar el equilibrio jurídico y político logrado en 1959 con la suscripción del Tratado Antártico, que ha permitido la cooperación de Estados con sistemas e intereses distintos y a veces contrapuestos, a la vez que la superación de problemas extremadamente difíciles y el establecimiento de precedentes valiosísimos en los campos del desarme y la investigación cientifica. 
6. Es dentro de la perspectiva que se ha procurado presentar, cómo debe estudiarse, a mi juicio, el tema apasionante al que se dedica el presente número del "Law Review".

Dentro de ella, conviene, antes que nada, detenerse en lo que hemos denominado como el Sistema Antártico.

\section{EL SISTEMAA ANTARTICO}

7. Puede sostenerse, con toda propiedad, que existe un Sistema Antártico, como lo ha desarrollado un eminente autor ${ }^{2}$.

En. efecto, durante el lapso de dos decenios, la Antártica ha sido eficazmente administrada bajo la tuición del Tratado suscrito en Washington en 1959 y las medidas adoptadas por las Partes Consultivas del mismo, en conformidad a sus disposiciones.

Si bien el Tratado Antártico no tiene una adhesión universal, pertenecen a él la generalidad de los Estados que de una manera u otra hau expresado su interés en el área. El Tratado está abierto a la adhesión de todos los Miembros de las Naciones Unidas. Sus disposiciones $\rightarrow \mathrm{y}$ la actividad consiguiente de las Partes Consultivas - han contado a lo menos con la aceptación tácita de la Comunidad Internacional. Esta aceptación ha sido aún explicitada en determinadas ocasiones, como ha ocurrido recientemente en la Organización para la Agricultura y la Alimentación o FAO $^{3}$.

8. En conformidad al Tratado Antártico — según se desarrollará a continuación- sus Partes Consultivas han establecido en el área situada al sur de los sesenta grados de latitud sur, una especie de reserva ecológica, un laboratorio de investigación científica, la primera zona desmilitarizada y desnuclearizada que ha conocido la Humanidad y una región de intensa y eficaz cooperación internacional en los más variados campos.

El subsistema antártico - que es parte del sistema internacional global- puede estimarse, en muchos aspectos, como más eficaz que este último. La administración de la Antártica, atendidas sus especiales características de región extrema y difícil, ha sido y es notablemente eficaz.

9. La cooperación en los campos de la investigación científica, la desmilitarización, la protección del medio ambiente, la meteorolo-

"The Antarctic System", Roberto E. Guyer, A. W. Sijthoff, Leyden, extract from the "Recueil des Cours", Volumen II, 1973, Academy of International Law

${ }^{3} \mathrm{La}$ Conferencia de la FAO celebrada en noviembre de 1975 "tomó nota de la competencia de las Partes del Tratado Antártico en todas Ias cuestiones relativas aI ecosistema de la Antártida" (citado en el Informe del XI Perfodo de Sesiones del Comité de Pesca o cofI de 1977, Documento FID/R 196 (Es). 
gía, la cartografía, las comunicaciones, el turismo y, en general, la administración de la inmensa área cubierta por el Tratado Antártico, pueden estimarse como constitutiva de un régimen jurídico expresado en el Tratado mismo, las medidas adoptadas en sus veinte años de vigencia, los derechos sobéranos a que se aludirá en su oportunidad y las actividades antes indicadas.

Se ha dicho que el Tratado no se refiere a los recursos antårticos, lo que es sólo parcialmente cierto. En efecto, aparte del Artículo rv y los derechos soberanos que cautela, el Artículo Ix, 1, letra f), incluye entre las medidas que deberán adoptar las Partes Consultivas, la "preservación y conservación de los recursos vivos de la Antártica". En obedecimiento de tal disposición, se han adoptado medidas para la protección de la fauna y flora, una série de disposiciones destinadas a proteger los recursos vivos en gèneral y se han originado dos Convenciones relativas a la conservación de los mismos: La Convención para la Conservación de las Focas Antárticas (Londres 1972); y la que considerará; sobre recursos vivos marinos en general, una Reunión Consultiva seguida de una Conferencia Diplomática programadas para la primera mitad de 1979 en Canberra ${ }^{4}$.

Se han manifestado dudas respecto a la extensión y autosuficiencia de los poderes mencionados con respecto a los recursos vivos en general. En todo caso, es cierto que el Tratado nada dispone sobre recursos minerales y que caben varias interpretaciones en cuanto a cómo debe procederse a su respecto.

Estas limitaciones no bastan, sin embargo, para desnaturalizar el régimen jurídico establecido y desarrollado por el Tratado Antártico ni afectan la existencia de lo que hemos denominado.el Sistema Antártico.

10. Para un mejor estudio del Sistema Antártico, convendría detenerse brevemente en la negociación y contenido del Tratado de Washington, en primer lugar; y tratar separadamente lo obrado por las partes activas del Tratado durante sus casi veinte años de vigencia.

\section{Negociación del Tratado Antártico}

11. En el trabajo introductorio se ha aludido al origen del Tratado Antártico, materia que requeriría probablemente un mayor

\footnotetext{
"Ver recomendación Ix, 2, de la Novena Reunión. Consultiva celebrada en Londres en 1977 ("Report of the Ninth Consultative- Meeting", London, - 1977) e Informes de la Primera y Segunda Etapas de la Segunda Reunión Consultiva Especial (Camberra, 27 de febrero al 16 de marzo de 1978; $y$ y Buenos Aires; 17 al 28 de julio de 1978).
} 
desairollo. Sin embargo, no será posible extenderse en esta oportunidad sobre tan interesante e importante materia ${ }^{5}$.

Es sabido que en 1958 culminó un gigantesco esfuerzo cooperativo internacional de conocimiento del Continente Antártico, que fue denominado "Año Geofísico Internacional" (IGY). En él participaron científicos de doce naciones, que ya tenían actividades y/o invocaban derechos en la Antártica y se interesaban especialmente por ella, muchas de las cuales instalaron bases o ampliaron las que ya habian emplazado en su territorio.

EI Año Geofísico Internacional evidenció la necesidad de mantener e incentivar la investigación científica. Dio ocasión, además, para iniciar una negociación que asegurase también la consagración de la Antártica como una zona de paz, superase ciertos conflictos de soberanía manifestados en el área, fomentase actividades deseables en ese Continente y preservase su ecología privilegiada.

El Gobierno de los Estados Unidos abrió esa negociación en 1958, sugiriendo la suscripción de un Tratado e invitando a participar en una serie de reuniones en Washington a los doce Estados que habian tomado parte en el IGY (Argentina, Australia, Bélgica, Chile, Francia, Japón, Nueva Zelandia, Noruega, la Unión Súdafricana, la Unión de Repúblicas Socialistas Soviéticas, el Reino Unido de Gran Bretaña e Irlanda del Norte, y los Estados Unidos de América).

12. El Tratado Antártico fue suscrito en Washington el $1^{\text {o }}$ de diciembre de 1959 por los doce países antes mencionados, cuyos nombres aparecen en el preámbulo de ese instrumento.

Los doce Estados suscriptores pasaron a constituirse en Partes Consultivas del Tratado, encargadas, en conformidad a su Artículo Ix, de recomendar medidas para el desarrollo de sus objetivos en el área del mismo.

En 1977 fue incorporado, como decimotercera Parte Gonsultiva, el Gobierno de Polonia, luego que una Reunión Consultiva Especial estableció que había reunido los requisitos necesarios para adquirir tal calidad.

El Tratado entró en vigor en junio de 1961, una vez depositado el último instrumento de ratificación.

Ver Tessup and Taubenfeld, obra citada; "U. S. Congress, Senate, Committee on Foreign Relations, The Antarctic Treaty", June 1960, Government Printing Office, Washington; y sobre todo, la obra recientemente publicada por el Profesor Enrique Gajardo, quien suscribió el Tratado Antártico por Chile: "Participación Chilena en la Elaboración del Tratado Antártico", Editorial Juridica de Chile, 1978. Esta ultima obra arroja nuevas luces sobre la preparación de la negociación de 1958 en los diez años precedentes y contribuye también al estudio de las reclamaciones de soberanfa. 
Han adherido al Tratado Antártico Brasil, Checoslovaquia, Dinamarca, Holanda, la República Democrática Alemana y Rumania.

13. La negociación del Tratado Antártico puede considerarse como una de las más significativas y exitosas de la historia contemporánea.

Ella logró reservar todo un Continente para la investigación científica y la protección ecológica, creando a la vez la primera zona desmilitarizada y desnuclearizada en el mundo.

Simultáneamente, se logxó superar el problema suscitado por las reclamaciones de soberanía en el Continente Antártico, protegiendo los derechos de los reclamantes, la posición de los no reclamantes y preceptuando que no se admitirian nuevas reclamaciones durante la vida del Tratado (Artículo IV).

En la negociación se obtuvo el acuerdo y la cooperación de paises representativos de sistemas políticos diferentes y aún opuestos, a lo menos en la época; de diversos grados de desarrollo; de posiciones jurídicas contrapuestas en lo que atañe a derechos sobre el. Continente Antártico y sus aguas; y representativos, finalmente, de los más diferentes sistemas jurídicos.

Por último, el resultado alcanzado con el Tratado de Washington, además de superar las dificultades suscitadas en el área por la guerra fría, dio origen al Sistema Antártico y permitió, así, una administración eficiente y ordenada de la Antártica.

14. En cuanto a la compatibilidad y armonía del Sistema creado por el Tratado con la Carta de las Naciones Unidas y la Comunidad Internacional en general, cabe acotar que él desarrolla los Principios y Propósitos de la Carta y puede estimarse como una expresión de los Acuerdos Regionales consagrados por la misma.

Lo obrado por el Tratado ha servido de precedente para otras zonas desnuclearizadas y de paz, lo que ha sido reconocido por el propio Secretario General de las Naciones Unidas en algunas de sus Memorias presentadas a la Asamblea General.

EI Tratado está abierto a la adhesión de todos los Miembros de las Naciones Unidas, sin condiciones de ninguna especie. Alienta, asimismo, el establecimiento de relaciones de carácter cooperativo con las Agencias Especializadas de las Naciones Unidas y otras organizaciones internacionales que tengan un interés científico o técnico en la Antártica (Artículo III).

Esta cooperación ha sido muy activa dentro de su área de competencia, con organizaciones tales como la Gomisión Oceanográfica Intergubernamental de la UNESCO (COI O IOC). La Organización Meteorológica Mundial (ONM O WMO), la Organización Mundial de 
la Salúd (oxis o who), la Organización Mundial de Tèlecomúnicaciones (OMT), el Programa de las Naciones Unidas para el Medio Ambiente (PNUMA o UNEP), la FAO, el SGAR, etc.

La negociación dió por resultado, en consecuencia, la creación de un subsistema perfectamente integrado dentro del sistema general de las Naciones Unidas.

\section{El sistema en la práctlca.}

15. A fin de poder evaluar y analizar in aplicación al área del Tratado Antártico de los principios del mismo conviene, en primer lugar, echar una mirada sobre su realidad actual.

16. En la Antártica existen alrededor de cincuenta bases científicas de carácter permanente, que aseguran no sólo el conocimiento profundizado y progresivo del medio, sino también las comunicaciones y la logística en esta área remota, en la que imperan condiciones extremas.

Año a año varios de los Estados que son Partes Consultivas llevan a cabo expediciones de carácter científico que amplían la labor permanente a que se ha hecho referencia y contribuyen a los variados aspectos de la presencia del hombre en el continente helado.

Las Partes Consultivas han instalado una red de comunicaciones que se integra al sistema universal y vincula el área antártica con el resto del planeta, facilitando, a la vez, las comunicaciones generales a través de este continente.

Existen estaciones meteorológicas, integradas al sistema unversal.

A trayés de la acción de los Miembros Activos del Tratado ha sido posible establecer un sistema de transporte terrestre, naval y aéreo, que aunque limitado permite el conociminto y la actividad del hombre en la zona. Estas instalaciones facilitan el estudio de la glaciología, las profundidades marinas, las realidades del medio ambiente y los organismos en la Antártica, todos ellos desarrollados al amparo del sistema establecido por el Tratado.

En la Antártica no existe contaminación apreciable; no se ha permitido la introducción en el medio ambiente de especies extrañas a él; se ha protegido la cadena biológica y las especies antárticas, configurando así lo que con justicia se ha denominado como una reserva ecológica en beneficio de la Humanidad. Esta realidad - a lo menos en dimensiones continentales-, es única en el mundo.

Finalmente, existe en la Antártica un conjunto de sitios especialmente protegidos y de áreas declaradas de especial interés científico; se aplican medidas para la protección y mantenimiento de los sitios y monumentos históricos, que son mudos testigos de la exploración 
y a veces del heroísmo de los hombres que penetraron en estas áreas remotas; se orienta y regula el turismo, de forma que no perjudique a la ecología.

17. Este conjunto de actos y actividades que representan una verdadera administración de la Antártica en beneficio de la Humanidad $y$ de conformidad con los objetivos antes señalados, ha sido logrado a través de la adopción por las Partes Consultivas de las medidas contempladas en el Artículo $\mathrm{xx}$ del Tratado y su implementación en el terreno.

Se ha disipado, así, el escepticismo que suscitó en un primer momento el sistema de recomendaciones y medidas, sin una autoridad central, que estableció el Tratado Antártico. La cooperación entre las Partes ha funcionado con tal expedición - tanto en la adopción de las recomendaciones unánimes como en su ejecución mismaque se ha logrado, como queda dicho, una eficiencia bastante inusuale. $^{\circ}$.

Todo ello ha supuesto, evidentemente, un esfuerzo nacional muy intenso de parte de los Estados fundadores y administradores del sistema y, especialmente, en el caso de algunos de ellos.

18. Las actividades de investigación científica se iniciaron con anterioridad al Tratado mismo; fueron intensificadas, alentadas $y$, podría decirse, sistematizadas por él.

19. La desmilitarización y desnuclearización de la Antártica han sido celosamente respetadas por las Partes del Tratado desde el primer instante de su vigencia, y aseguradas y cauteladas por el sistema de inspección establecido en el instrumento mismo.

Estas realidades superaron los temores derivados de una competencia política y militar latente al momento de la negociación del Tratado, estableciendo a la vez un precedente de importancia en el campo del desarme.

\section{Protección del Medio Ambiente y Conservación de los recursos}

Estos objetivos han consi tuido una de las preocupaciones centrales de las Partes activas del Tratado Antártico, tanto en el estudio y adopción de Medidas a su respecto como en su implementación en el área del Tratado.

La preocupación por estos temas se manifiesta desde la Primera Reunión Consultiva del Tratado, pero culmina en la Tercera, celebrada en Bruselas en 1964, que adopta las "Medidas Convenidas

-Ver Guyer, obra citada, Capítulos III y Iv. 
para la Protección de la Fauna y Flora", conocidas también, más simplemente, como "Medidas Convenidas"7.

La primera $\rightarrow$ y fundamental- de estas medidas, destinadas a la protección de la ecología antártica, es la declaración del área de aplicación del Tratado como "Un aérea especial de conservación". (Terminología que supone una prioridad terminante de la consideración ecológica respecto de cualesquiera actividades desarrolladas al sur de los 60 grados de latitud sur).

21. Las Medidas Convenidas aseguran la protección de todas las especies nativas, sean mamíferos o pájaros, y adoptan disposiciones extremadamente restrictivas respecto a la posibilidad de su captura o muerte, que son sometidas a un riguroso sistema de permisos (artículo vI).

También protegen a todas las especies de interferencias dañinas que alteren de cualquier manera sus condiciones normales de vida (artículo vir).

Contemplan la creación de Areas Especialmente Protegidas. y Sitios de Especial Interés Científico, para Ios cuales se prohiben aún actividades consideradas legítimas en otros sitios de la Antártica, a fin de preservar la pureza ecológica de determinados medios y especies o permitir una investigación científica de notable interés. Las Reuninones Consultivas siguientes han declarado, modificado, suprimido y añadido muchos sitios especialmente protegidos.

22. El desarrollo, complementación y cumplimiento de estas medidas ha descubierto Ia sucesión bianual de Reuniones Consultivas y dirigido la actividad en el área de los países antárticos.

23. EI complemento más importante de este conjunto de medidas de conservación de los recursos y preservación de la ecología ha sido el estudio y adopción de medidas respecto al "Impacto del hombre en el Medio Ambiente Antártico".

La vi Reunión Consultiva celebrada en Tokio en 1970, reconociendo que "el ecosistema antártico es especialmente vulnerable a Ia interferencia humana" y que existe urgente necesidad de proteger el medio ambiente antártico de dicha interferencia, decide que las Partes Consultivas asuman responsabilidad por su protección. Al efecto, invitan al Comité Científico sobre "Investigación Antár-

Ver ANTARTIC TREATY," "Handbook of Measures in Furtherance of the Principles and Objetives of the Antarctic Treaty", September 1977, London, página 2101. Esta obra, preparada con ocasión de la Ix Reunión Consultiva, celebrada en Londres en 1977, contiene una recopilación por temas de las Medidas adoptadas por el Sistema Antártico.

Ver -en la misma materia- Guyer, ob. citada, páginas 193 y siguientes. 
tica o SCAR a que identifique los tipos de interferencia humana, evalúe los posibles cambios en el Medio Ambiente y recomiende medidas 8 .

Sobre la base del informe del SGAR, la viII Reunión Consultiva -reunida en Oslo, en 1975- adoptó un Código de Conducta para las bases y actividades en la Antártica en general. La misma reunión, reconociendo que "la responsabilidad primordial en asuntos antárticos, incluyendo la protección del medio ambiente antártico corresponde a los Estados activos en el área que son Partes del Tratado Antártico, formuló un recuento de las medidas de protección ecológicas adoptadas por las Partes Consultivas y recomendó nuevas medidas. El texto de la recomendación virr-13 es digno de ser consultado?.

24. El tema del Impacto del Hombre sobre el Medio Ambiente Antártico fue considerado en relación con las cuestiones relativas al aprovechamiento de los recursos vivos y minerales tanto en la virr como en la Ix Reunión Consultiva del Tratado, celebradas respectivamente en Oslo (1975) y Londres (1977).

Como se verá más adelante, las Recomendaciones de la última Reunión Gonsultiva ordinaria (Londres, 1977) así como el anteproyecto de Convención sobre conservación de los recursos marinos antárticos, contemplan una clara prioridad ecológica sobre cualquier actividad comercial de aprovechamiento de los recursos.

Además de esa prioridad, la Reunión Consultiva de Londres reiteró la vinculación del ecosistema antártico con los ecosistemas vecinos, relacionados con o dependientes de él. En efecto, la Recomendación IX-5, sobre Impacto del Hombre en el Medio Ambiente, declara que "Las Partes Consultivas asegurarán en el planteamiento de futuras actividades que la cuestión de los efectos ambientales y el posible impacto de tales actividades en los ecosistemas pertinentes sean debidamente considerados"10. Una mención semejante puede encontrarse en los "principios" que deberán orientar un posible régimen para el aprovechamiento de los recursos minerales antárticos, cuando expresan que "Ia protección del singular ambiente antártico y de sus ecosistemas dependientes debe ser una consideración básica" (Recomendación IX-1) ${ }^{11}$. El informe de la IX Reunión Consultiva, al tratar del Impacto del Hombre sobre el Medio Ambiente, comprueba que en conjunto con SCAR las Partes Consultivas "han desarrollado planes para el estudios comprensivo del ecosiste-

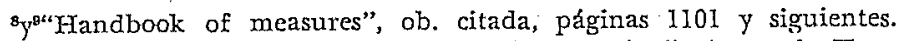

IoVer "Report of the Ninth Consultative Meeting". Antarctic Treaty. London, 1977 , pág. 18.

I1Ver "Report of the Ninth Consultative Meeting", ya citado, páginas II $\mathrm{y}$ siguientes. 
ma marino antártico". A este respecto deberán "identificar los tipos y extensión de la interferencia humana en el medio" y "evaluar el posible impacto sobre el Medio Ambiente del área del Tratado y otros ecosistemas dependientes si la explotación mineral fuere a tener lugar alli".

Se deduce de todo lo anterior que las Partes Consultivas del Tratado Antártico han decidido que cualquier aprovechamiento comercial futuro de los recursos contenidos en el área del Tratado no debe afectar el ecosistema antártico y los ecosistemas relacionados con él.

Estos ecosistemas son los correspondientes a las regiones más cercanas al continente antártico.

25. La relación entre la protección ecológica y el posible aprovechamiento de los recursos fue expresada por las Partes Consultivas del Tratado Antártico en un texto que convendría reproducir en su integridad, en su versión inglesa:

"With the items on the agenda concerning Antarctic resources "particularly in mind, the Representatives discussed the question "of man's impact on the Antarctic environment. They recalled "the numerous steps already taken by Consultative Parties "designed to protest the Antartic environment from unnecessary "interference, including:

"(i) The designation by the Consultative Parties of the "Treaty Area as a Special Conservation Area and the adoption "of the "Agreed Measures for the Conservation of the Antarctic "Fauna and Flora";

"(ii) The designation of "Specially Protected Areas" to "preserve their unique ecological system and "Sites of Special "Scientific Interest" to enable scientific investigations to be .. "carried out at the those sites without inteferences;

"(iii) The negotiation of the Convention for the Conservation "of Antarctic Seals (London 1972);

"(iv). The adoption of measures to preserve and protect "from damage historic monuments in the Antartic Treaty Area; "(v) The adoption of a Code of Conduct to be observed at "their stations and by their expeditions within the Antarctic "Treaty Area.

They also recalled that in close co-operation with "the Scientific Committee on Antarctic Research (SCAR) of the "International Council of Scientific Unions, and through SCAR "with other appropriate international organizations concerned, "they have developed plans for the comprehensive study of the "Antartic marine ecosystem considered as an integral part of "the Antarctic environment and have sought to: 
(a) identify the types and assess the extent of human interferance which has occurred in the Treaty Area as a result of man's activities;

(b) assess the possible impact on the environment of the Treaty Area and other dependet ecosystems if mineral exploration and/or exploitation were to occur there.

The Representatives, while considering the next "steps to be taken with regard to questions concerning Antarctic "resources, decided to recommend that their Governments should "reaftirm their commitment to environmental protection.

"Accordingly the Representatives drew up the statement contained "An Recomendation $1 \mathrm{X}-\mathrm{b}$ ".

26. La preocupación ecológica se basa en estudios científicos de la más variada indole, llevados a cabo generalmente por especialistas de los miembros activos del Tratado Antártico o en programas organizados bajo el patrocinio de las Partes Consultivas o estimulados por ellas.

Hemos hecho referencias a los estudios de SGAR y, especialmente, a los que dicen relación con el Medio Ambiente Antártico. Cabe destacar - como presentación del ecosistema marino antártico- el Programa llamado "Bromass", iniciado a requerimiento del Sistema Antartico e impulsado por SGAR, en colaboración con scor (Scientitic Committee on Ocean Research) y FAO.12

Se ha establecido cientificamente el carácter único de ecosistema antártico; su influencia en el clima del mundo, en la reserva global de agua dulce, en las corrientes, en la formación de zooplancton; la existencia de un ecosistema marino antártico, circunscrito por la "convergencia antártica" y relacionado con el Medio Ambiente terrestre del continente y con las ecologías vecinas a él y situadas más allá del llamado "Océano Austral".

También ha podido dimensionarse en principio el riesgo que pociría significar una explotación indiscriminada de los recursos vivos en la Antártica y, especialmente, del "krill", pequeño crustá. ceo que parece ser uno de los ejes de su ecosistema marino ${ }^{13} ; y$, en

1Biological Investigations of Marine Antarctic System and stocks". Ver estudio presentado por el Programa BIOMAS a la Ix Reunión Consultiva de Londres en 1977 y a la Comisión Oceanográfica Intergubernamental (CoI)
de la uNESco el mismo año. Ver doc. CoI / INF. 337, de 1977. Informes $\mathrm{x}$ Periodo cor y XI Período coFr.

IBEversen, "The Living Resources of the Southern Ocean." FAo Southern Ocean Survey Programme, GLo/so/77/1; Richard M. Laws, "The Antarctic Marine Ecosystem", London, 1978. 
Io que toca a una posible explotación mineral, el peligro serio que podría presentar para el Medio Ambiente ${ }^{14}$, ${ }^{15}$.

La competencia de las Partes Consultivas sobre todo lo relacionado con el ecosistema antártico fue reconocida, como queda dicho, por la Conferencia de la FAO celebrada en 1975.

27. Fluye de todo lo anterior la importancia que deberá atribuirse a la preservación del Medio Ambiente singular de la Antártica, a la preservación y conservación de sus recursos y a la mantención del equilibrio de su ecosistema y de los ecosistemas vecinos.

28. Estudiado en forma muy general lo que pudiéramos liamar "el factor ecológico", convendría, probablemente, antes de abordar la cuestión de los recursos en la zona, detenerse en el problema de la soberanía, que ha sido tratado en el estudio preliminar que abre la consideración del tema en este "Journal of Law".

El problema de la soberania.

29. La discusión del tema del ałrovechamiento de los recursos antárticos -al que nos referiremos más adelante-, plantea el problema de la soberanía, que es parte de lo que hemos denominado el Sistema Antártico.

Se trata de determinar qué validez y alcance tienen las reclamaciones de soberanía formuladas por siete Estados (Argentina, Australia, Ghile, Francia, Noruega, Nueva Zelandia y Reino Unido), sobre la casi totalidad del continente antártico; y qué efecto tiene - puede tener la soberanía invocada sobre los espacios marítimos (Mar Territorial, Plataforma Continental, Zona Económica) que conforme al Derecho Internacional general son consecuencia necesaria de la soberanía terrestre.

30. El Tratado Antártico no resolvió el problema de las reclamaciones ni tampoco, como se ha dicho, "congelo" a aquél o a éstas. Lo que hizo - simplemente- fue mantener el "statu quo".

Su artículo IV preceptúa, en su primera parte, que nada en él puede interpretarse como renuncia a derechos previamente hechos valer o reclamaciones de soberanía territorial en la Antártica; ni

\footnotetext{
${ }^{14}$ Report from the Ninth Consultative Meeting, ya citado. Referencias al informe de SCAR e Informe del Grupo de Expertos sobre exploración y explotación mineral, Anexo v, pág. 56.

${ }^{25}$ Resultados del Simposium Internacional sobre Desarrollo de la Antártica que tuvo lugar en Punta Arenas, Chile, en el Verano Austral de 197\%, con participación de muchos de los expertos mundiales. Publicaciones del Instituto de Estudios Internacionales de la Universidad de Chile, 1977.
} 
tampoco como prejuzgando la posición de quiénes no reconocen tales derechos.

Protege, así, el Tratado los derechos hechos valer por los reclamantes; y la posición de los no reclamantes.

Agrega que las actividades desarrolladas durante la vigencia del Tratado no podrán mejorar o perjudicar las reclamaciones; y que no podrá extenderse las fechas o formularse nuevas en vida deI Tratado.

31. En el fondo, el problema de la soberanía no se presentaba ni presentará con claridad hasta que se plantee definitivamente el aprovechamiento económico de los recursos.

El Tratado Antártico no trató propiamente esta cuestión, si bien se refirió a la conservación de los recursos vivos como a una de sus funciones esenciales y preservó los derechos y posición invocadas respecto a la soberanía. La reserva ecológica, el laboratorio cientifico y la zona de paz constituían, de hecho, una especie de moratoria.

Planteado el problema de los recursos, revive la situación de discrepancia planteada en el artículo IV.

32. No es el momento ni la ocasión de plantear a fondo el tema de la validez general y particular de las reclamaciones. Siguiendo el llamado de la revista - y atendidos la naturaleza general y preliminar de este artículo y el apremio del tiempo-, no desarrollaremos propiamente este asunto, si bien nos sentimos obligados a formular algunos comentarios.

33. No cabe duda de que nada. en el Derecho Internacional impide que haya soberanía sobre las tierras polares y derechos soberanos sobre las aguas y Plataformas Continentales adyacentes a ellas, estén o no cubiertas las unas y las otras por hielos. Esto se desprende con toda claridad del Derecho Internacional general y ha sido confirmado por muchos tratadistas ${ }^{16}$.

Tampoco cabe duda de que será necesario acreditar el equivalente de la dualidad que en Derecho Privado se expresa en la concurrencia del título para la adquisición del dominio y la posesión. Este último término ha sido reemplazado por el de "ocupación efectiva", en muchos comentarios relacionados con esta cuestión.

34. En los planteamientos formulados en algunas ocasiones, $y$ en

${ }^{20} \mathrm{La}$ IIr Conferencia de las Naciones Unidas sobre Derecho del Mar no ha elaborado normas de excepción para tierras o aguas heladas en esta materia. Ver el Texto Integrado de Negociación, Doc. A/CONF. 62/WP. 10 y Add I. 1977. 
parte hechos valer en este número, se analiza la situación con una perspectiva que podría ser estimada como de preguerra $y$, en ocasiones, con un cierto exclusivismo de enfoque jurídico de carácter anglosajón.

El distinguido Profesor francés Rene Dupuy planteaba que en el caso de las reclamaciones de sóberanía en la Antártica existía, en el caso del título invocado, una confrontación de contigüedad y descubrimiento ${ }^{17}$. En efecto, los países vecinos a la Antártica y, en especial, los más cercanos a ella (Chile y Argentina) invocan la continuidad y contigüidad, en tanto que aquellos Estados que participaron más extensamente en una época en la exploración del continente, acentúan el descubrimiento ${ }^{18}$.

La contradicción descrita es, sin embargo, sólo la segunda parte del problema. En efecto, debe determinarse - primero-, si se invoca un tỉfulo originario sobre la Antártica o uno derivativo o, en otras palabras, si la Antártica ha sido o es en todo o en parte "res nullius".

35. Chile y Argentina invocan un título originario, derivado de los derechos de la Corona de España, en virtud del Tratado de Tordesillas, a lo que en la época se denominaba como "Terra Australis"18. Este derecho, a lo menos en el caso chileno, fue estimado. como digno de consideración por el eminente tratadista Gilbert Gidel19.

Los derechos españoles pasaron a sus ex colonias cuando alcanzaron la independencia, en 1810, en virtud de lo que se ha denominado como el "utti possidetis juris". En el caso de Chile, ellos fueron reafirmados - con expresa referencia a la Antártica- por el primer gobernante de su era independiente y afianzados por los actos ejecutados en el Estrecho de Magallanes hacia el Sur, con asiento principal en la cirudad de Punta Arenas ${ }^{20}$.

17"Le status de "L'Antarctique", Annuiare Français de Droit International, 1958, páginas 208 y siguientes.

${ }^{18} \mathrm{El}$ argumento de la continuidad adquiere especial forma y significación cuando se trata de países situados a no más de 500 millas de la Antártica, como es el caso de Chile.

(18 bis) Oscar Pinochet de la Barra, "Chilean Sovereignty in Antarctica", Editorial del Páfífico, Santiago, 1954. El título chileno deriva de una concesión a don Pedro Sánchez de la Hoz, a quien se confirió la Gobernación las tierras al sur del Estrecho de Magallanes hasta el Polo Sur en 1539 Esta concesión pasó a la Capitanía General de Chile por Reales Cédulas de 1555 y 1558, que las incluyeron en las Gobernaciones de Jerónimo de Alderere y Francisco de Villagra (ver páginas 14 y 15 , obra citada).

20"Aspects juridiques de la lutté pour L'Antarctique", París, 1948, página 55.

${ }^{30}$ Oscar Pinochet de la Barra, obra citada; Armando Braun Menéndez, "Pequeña Historia Antártica", Editorial Francisco de Aguirre, Buenos Aires, 1976. 
A principios de siglo, Chile otorgó concesiones de pesca y caza en la Antártica, en 1902 y 1906. En este último año, además, se instaló en la isla Decepción, de las islas Shetland del Sur, "La Compañía Ballenera de Magallanes", sociedad chilena que operaba con un permiso otorgado por el Gobernador de Magallanes ${ }^{21}$. También en 1906, el Ministro de Relaciones Exteriores de Ghile alude públicamente a los derechos antárticos de su país e inicia una negociación con Argentina de delimitación antártica, que se extiende a $1907^{22}$.

Es, por tanto, en 1906 cuando estiman autores chilenos que el título de ese país fue perfeccionado ${ }^{23}$. Al dictarse el Decreto de 1940, que fijó los límites del territorio chileno antártico, éste dejó constancia de la preexistencia de los derechos invocados y los actos de soberanía de $1906^{24}$.

Los fundamentos de los derechos invocados por Chile han sido circunstancialmente analizados en la obra del Doctor Pinochet de la Barra, tantas veces citada. No es nuestro propósito tratarlos a fondo en este artículo 24 bis.

36. Si se invoca un título originario, con algún fundamento, las invocaciones coadyuvantes a la continuidad y la contigüidad se refuerzan grandemente.

Se ha dicho - por algunos autores- que sólo el descubrimiento puede ser base de dominio sobre territorios extremos. La verdad es que esta noción debe su extensión y popularidad a la época del colonialismo, en la que fue invocado como fundamento de dominio en grandes extensiones de Africa y Asia.

Para su aplicabilidad a esta situación, sería necesario, en todo caso, que ningún Estado hubiere acreditado título o presencia con anterioridad; si se presentase un "res nullius" cumplido y total.

Los casos ventilados ante Tribunales Internacionales y frecuentemente citados con relación a territorios escasamente habitados y la soberanía a su respecto son todos anteriores a la gran guerra, sin que ésto prive a algunas de sus consideraciones de validez. $\mathrm{Ha}$ habido, entretanto, un "desarrollo progresivo del Derecho Internacional", en el sentido preciso que le da la Carta de las Naciones Unidas.

2xPinochet, obra citada, página 38; Braun Menéndez, obra citada, páginas 123 y siguientes.

Pinochet; obra citada, página 57.

${ }^{3}$ Pinochet, obra citada, página 57.

2Pinochet, obra citada, páginas 53 y 54.

(\% bis) Cabe recordar que Chile y Argentina estiman su reclamación antártica como parte de sus respectivos territorios, a la que pueden considerarse como juridica y físicamente unidos. Ambos países se han reconocido mutuamente derechos en la Antártica en numerosas ocasiones. 
37. La evolución del Derecho favorece evidentemente, en el caso de las reclamaciones antárticas a aquellos reclamantes cuyo territorio está muy cerca de la Antártica y vinculado a él por factores geográficos, ecológicos, económicos y aun estratégicos. En el caso de Chile, como lo sostiene un destacado autor francés, citando el testimonio del Almirante Byrd, "los recientes trabajos científicos parecen demostrar que la Península Antártica es ni más ni menos que una extensión de la América del Sur y que las dos regiones pertenecieron otrora a un único Continente cuyas partes hoy. sumergidas constituyen la plataforma submarina más acá y más allá del Estrecho de Drake"25.

38. En relación a los argumentos basados en la continuidad y la contigüidad, se ha criticado la llamda Teoría del Sector o prolongación geométrica hasta el Polo de un territorio vecino.

Sin que esto signifique cuestionar los méritos de dicha teoría, podría estimar que ella admite algunas críticas que son justificadas. Una de ellas, en el caso de la Antártica, sería que los sectores comprenderian aguas que forman parte de la alta mar.

A este respecto, y en lo que toca a Chile, el Decreto de 1940 que precisó los límites antárticos del país alude al Mar Territorial dentro del cuadrante reclamado, lo que presupone la existencia de la alta mar más allá de las 200 millas jurisdiccionales declaradas por ese país en $1947^{25}$ bis.

39. En cuanto a la posesión u "ocupación efectiva", las consideraciones formuladas en los casos tantas veces citados por los autores y relativos a territorios extremos, tienen plena validez.

El ejercicio de la autoridad, de actos de administración y de actividades económicas bajo el control de un Estado, debe entenderse adaptado a las condiciones geográficas y climáticas de la Antártica.

Es en esa perspectiva que deben analizarse los actos de administración, instalación de bases, actividades económicas y científicas de los países reclamantes, llevados a cabo antes de la suscripción del Tratado Antártico ${ }^{26}$, sobre los que no profundizaremos en esta oportunidad.

2J. F. da Costa, "Souveranaité sur L'Antarctique" obra citada, página 2. Ver también las referencias al Tratado Interamericano o TuAR (Río de Janeiro 1974), que incluyó en la zona de defensa del Continente el conjunto de las reclamaciones de Chile y Argentina.

( ${ }^{35}$ bis) En el caso de Chile, sus actos de administración se remontan, en este siglo, a los comienzos de 1902 y 1906, la vinculación en la Provincia de Magallanes; la presencia de numerosas bases previas al Año Geofísico Internacional y al Tratado Antártico; la emisión de sellos, etc. Su presencia económica se ha manifestado en las industrias de la foca, la ballena y el "krill", entre otras,

${ }^{26}$ Ver Guyer, Gajardo, Pinochet, Jessup y Da Costa, obras citadas. 
40. En consecuencia, será preciso, al evaluar el mérito de las reclamaciones, tener en cuenta los títulos históricos y la contigüidad -no sólo el "descubrimiento"- en conjunto con la posesión u ocupación efectiva.

41. En cuanto a Ios Espacios Marítimos -Mar Territorial, Zona Económica y Plataforma Continental-, son una consecuencia del dominio terrestre. Una vez más, es menester comprobar que el Texto Integrado de Negociación o ante-proyecto de Convención preparado por la Tercera Conferencia de las Naciones Unidas sobre el Derecho del Mar no hace discriminaciones al respecto y se limita a enunciar la regla general, aplicable por consiguiente a todos los casos.

En el caso de los reclamantes, sólo uno de ellos había proclamado su jurisdicción sobre una zona de 200 millas aplicable a su reclamación antártica con anterioridad a la suscripción del Tratado de Washington ${ }^{27}$. Un segundo reclamante lo hizo ya hace algunos años ${ }^{28}$, en tanto que dos de ellos han dictado legislaciones que habilitan al Poder Ejecutivo para extender su Zona Económica de 200 millas a su reclamación antártica ${ }^{29}$.

Se ha sostenido que la dictación de nuevas zonas jurisdiccionales de 200 millas constituiría una extensión de las reclamaciones, en los términos de la parte final del Artículo xv del Tratado Antártico. Si se considera que los espacios marítimos no son sino una consecuencia natural y necesaria de la soberanía terrestre, tal argumento caería por su base.

También se ha dicho, por algunos, que todas las aguas de la Antártica serían Altar Mar. Tal iaterpretación se contradice, sin embargo, con el tenor mismo del Artículo vi del Tratado Antártico, que al aludir a la Alta Mar está manifestando, "a contrario sensu", que existe en el área del Tratado a lo menos un Mar Territorial ${ }^{30}$. Tampoco se compadece con el hecho, ya anotado, de que a lo menos uno de los firmantes originales de Washington se incorporó al Tratado con una reclamación que comprendía una zona jurisdiccional de 200 millas.

\section{Todo lo anterior llevai a la conclusión de que necesariamente}

${ }^{27}$ Chile, Declaración de Zona Naritima de 200 millas, en 1947.

${ }^{23}$ Argentina.

${ }^{2}$ Australia y Nueva Zelandia.

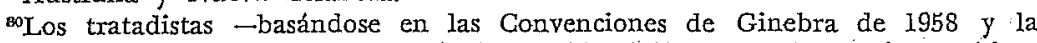
Costumbre Internacional- han definido la Alta Mar como el espacio marítimo situado más allá del Mar Territorial, Por tanto, la Alta Mar supone la existencia de una soberanía extendida a las aguas o Mar Territorial (en la actual Conferencia del Mar la Alta Mar comenzaría más allá de la Zona Económica de 200 millas). 
cualquier régimen de aprovechamiento de los recursos antárticos - minerales o vivos - tendrá que tener en cuenta la realidad de la afirmación de soberanía en la Antártica.

43. El propósito y la extensión limitada de este artículo nos han impedido tratar en profundidad la cuestión de las reclamaciones de soberanía en la Antártica. Las consideraciones que preceden deben ser entendidas como un bosquejo de algunos problemas y definiciones que estimamos necesarios en un análisis global del tema de los recursos antárticos.

\section{La cuestión del aprovechamiento de los recursos}

En la introducción se ha aludido al creciente interés suscitado en la última década por los recursos antárticos.

La llamada "crisis del petróleo" motivó, a partir de 1970, la búsqueda de nuevas fuentes de energía. En esa perspectiva, los Estados y grandes corporaciones, comenzaron a interesarse, entre otras posibilidades, en los hidrocarburos que pudieren existir en las Plataformas Continentales de la Antártica:

En un proceso simultáneo, como ya se ha relatado, la concentración de pesca en las regiones tradicionales y la universalización de las Zonas Económicas o de Pesca de 200 millas, produjeron un interés renovado y creciente por los recursos vivos de los mares australes. Este proceso se agudizó en 1974 con los progresos logrados en la Tercera Conferencia de las Naciones Unidas sobre el Derecho del Mar.

En los últimos años, se ha hecho presente una mayor urgencia en cuanto a la necesidad de un régimen para los recursos vivos, en tanto que la cuestión de los recursos minerales, conservando su importancia, no ha sido objeto, por diversos factores, del mismo grado de apremio.

45. Recursos vivos. El tema de los recursos vivos marinos de la Antártica fue presentado por Noruega a la Octava Reunión Consultiva celebrada en Oslo en 1975, la que acordó que làs Partes Consultivas se abocasen a esta cuestión y asumiesen respecto a la conservación de dichos recursos sus responsabilidades especiales ${ }^{31}$.

En tanto que el sistema del Tratado Antártico abordaba este delicado problema, el Comité de Pesca de la FAO O COFI estudiaba, a partir de 1975, el tema de los recursos marinos de los mares australes, situados al sur de $45^{\circ}$ de latitud sur.

La FAO reconoció - como queda dicho- la competencia especial de las Partes del Tratado Antártico en todo lo relativo a la 2501.

"Ver "Handbook of Measures", obra citada, recomendación vHI, 10, página 
preservación y conservación del ecosistema en el área del Tratado; afirmó su propia competencia técnica en lo que dice relación al estudio y conocimiento de los recursos vivos australes; expresó su voluntad de cooperar con las Partes Consultivas en dicha materia; dejó constancia del necesario respeto por las jurisdicciones costeras en los mares australes; formuló diversas apreciaciones técnicas respecto a la naturaleza de los recursos australes, especies, cantidades conocidas, actividades en el área, investigaciones, etc.; manifestó su aprecio y deseo de continuada colaboración con el Programa "BIOMASs", originadó por las' Partes del 'Tratado Antártico; y, finalmente, en su última reunión, celebrada en este año en Roma, dejó constancia de la cooperación que mantenía con las Partes Consultivas del Tratado $\mathrm{y}$ tomó nota de la preparación por éstas de un régimen para los recursos vivos ${ }^{32}$.

46. La Novena Reunión Consultiva del Tratado Antártico, celebrada en Londres en septiembre de 1977, consideró extensamente el tema de la conservación de los recursos vivos antárticos, siguiendo algunas reuniones preparatorias sobre la materia, y adoptó la Recomendación IX-233.

La recomendación indicada, luego de referirse a la investigación científica y a adoptar directrices tradicionales para la conservación de los recursos vivos, se refiere al "establecimiento de un régimen definitivo de conservación, en los siguientes términos:

"1. Que se establezca un régimen definitivo para la conservación de los recursos marinos vivos del Antártico antes de finales de 1978. "2. Que se convoque una reunión Consultiva Especial para elaborar un proyecto de régimen definitivo y, en particular, para:

a) determinar la forma de ese régimen definitivo, incluida la cuestión de si es necesario un instrumento internacional como, por ejemplo, un convenio;

b) preparar, en caso necesario, un proyecto de reglamento para una reunión decisoria posterior, en orden al establecimiento del régimen definitivo;

c) decidir sobre la participación en dicha reunión de Estados que no sean Partes Gonsultivas y participen activamente en la investigación y explotación de los recursos marinos vivos del Antártico y sobre la participación, en calidad de observadores, de las organizaciones internacionales correspondientes;

d) determinar la fecha y lugar de la reunión decisoria;

Informes del corr, correspondientes a sus sesiones de 1977 y 1978 . Ver documento FW/R. 196 (Es) y COFI 78/7;

"Ver "Report of the Ninth Consultative Meeting", ya citado, página $12 \mathrm{y}$ siguientes. 
e) tomar todas las demás medidas que sean necesarias para facilitar los trabajos indicados de la reunión decisoria.

3. La Reunión Consultiva Especial habrá de basar sus trabajos en esta recomendación y tener en cuenta los debates de la Novena Reunión Consultiva, su informe y los documentos presentados, $y$, a la hora de preparar un proyecto de régimen definitivo, habrá de tener en cuenta, entre otros, los siguientes elementos:

a) el régimen habrá de reconocer explícitamente las responsabilidades primarias de las Partes Consultivas en lo relativo a la protección y conservación del medio ambiente del área del Tratado Antártico y la importancia de las medidas recomendadas por las Partes consultantes a tal fin;

b) Io dispuesto en el Artículo 4 del Tratado Antártico no se verá afectado por dicho régimen, que habrá de asegurar que se salvaguarde la aplicación de los principios encarnados en el Capítulo 4 a las áreas marinas situadas al sur de los $60^{\circ}$ de latitud Sur;

c) el régimen debe incluir las disposiciones necesarias para Ia eficaz conservación de los recursos marinos vivos del ecosistema del Antártico en su conjunto;

d) el régimen debe abarcar el área de competencia específica del Tratado Antártico;

e) el régimen, sin embargo, ha de extenderse al norte de los $60^{\circ}$ Sur cuando ello sea necesario para la eficaz conservación de las especies del ecosistema del Antártico, sin perjuicio de la jurisdicción de los Estados costeros en esa zona;

f) el régimen no habrá de aplicarse a las especies ya reguladas por acuerdos internacionales vigentes, pero habrá de tener en cuenta la relación de esas especies con las incluidas en el régimen".

47. Como secuela de la recomendación que se ha reproducido, se ha celebrado durante 1978, en varias etapas, la Segunda Reunión Consultiva Especial del Tratado Antártico (reuniones en Canberra, en enero; en Buenos Aires, en julio; y Consultas Informales en Washington, en septiembre).

En dichas Reuniones se ha considerado la preparación de un Proyecto de Convención sobre la Conservación de los Recursos Vivos Antárticos, basado en la Recomendación Ix-2.

El Proyecto reconoce la responsabilidad primordial de las Partes Consultivas para la protección del medio ambiente antártico y la preservación $\mathrm{y}$ conservación de sus recursos vivos; consecuente. mente con lo anterior, define como su objetivo la conservación de todos los recursos del ecosistema antártico, estableciendo, para tales efectos, un área de aplicación que se extiende a la convergencia antártica y definiendo criterios o "standards" de conservación 
de la mayor estrictez; se refiere solamente a la conservación de los referidos recursos; establece, a tales efectos, una Comisión y un Comité Gientífico.

La Convención estaría abierta a todos los Estados, pero la participación en la Comisión exigiría ser miembro originario de la misma o actiro e materias de investigación o recolección de los referidos recursos.

El Proyecto aplicaría los principios del Artículo Iv del Tratado Antártico - relativo a las reclamaciones de soberanía- a las áreas marinas situadas al sur de los $60^{\circ}$ de latitud sur.

48. En este esfuerzo, las Partes Consultivas han actuado en consultai con los Estados activos en el área y con la FAO.

Su intención es completar la Convención propuesta en la primera mitad de 1979.

49. Se desprende de la sumaria exposición precedente que la responsabilidad de la preparación de un régimen para los recursos vivos de la Antártica ha sido asumida por las Partes Consultivas del Tratado, con la plena aceptación, como queda relatado, de la FAo y de otros interesados.

Es natural que asf sea, pues existe - como lo hemos sostenido largamente- un subsistema antártico, integrado al sistema internacional general y responsable de la administrạción de la Antártica.

La relación entre el aprovechamiento de los recursos y las medidas adoptadas bajo el sistema antártico para la mantención del Continente como un área especial de conservación y un laboratorio científico debe ser reconocida y mantenida en cualquier régimen. Así lo hace el Proyecto de Convención a que se ha hecho. referencia, que deberá expresar una serie de vínculos con el Tratado Antártico, entre los cuales cabe destacar su estricta mantención como zona de paz y el respeto de las medidas adoptadas para la protección del medio ambiente, junto al reconocimiento de la responsabilidad especial de las Partes Consultivas para la protección del ecosistema.

50. Recursos minerales. La cuestión de la posible exploración y explotación de los recursos minerales antárticos, junto a los efectos que ellas pudieren tener para la ecología de ese Continente privilegiado, fue mencionado, por primera vez, en la Sexta Reunión Gonsultiva celebrada en Tokio en 1970. Pero su consideración se hizo, en forma detenida, en la Reunión Consultiva celebrada en Wellington, en 1972 (Séptima). En esta Reunión se acordó seguir 
estudiando el tema $y$, en especial, los efectos que una posible explotación pudieren tener sobre el medio ambiente 34 .

La Octava Reunión Consultiva, celebrada en Oslo en 1975, consideró nuevamente el problema, reiterando y ampliando las conclusiones de Wellington, que comprendían una moratoria hasta el definitivo esclarecimiento del problema y la consiguiente adopción de un régimen o las correspondientes decisiones ${ }^{35}$.

Entre ambas reuniones, tuvo lugar la Reunión Informal de la Fundación Nansen, a que alude el trabajo introductorio de esta revista ${ }^{36}$.

51. La Novena Reunión Consultiva, celebrada recientemente en Londres en 1977, conoció un Informe del SGAR sobre los posibles efectos de una exploración mineral ( $y / 0$ explotación), con especial referencia al petróleo. Este informe señalaba los variados peligros que tal exploración y explotación pueden significar para el medio ambiente.

Un grupo de expertos, ampliando el Informe del SCAR, y otros estudios y desarrollando el tema durante el curso de la reunión misma, emitió un Informe sobre la materia, que deberá ser completado mảs adelante ${ }^{37}$.

52. La Reunión de Londres concluyó sus trabajos en la materia con una recomendación de la mayor importancia, preparada sobre la base de los ácuerdos de las Reuniones Consultivas anteriores y de una Reunión Freparatoria Especial celebrada en París en 1976. Esta Recomendación, en su texto inglés, dice textualmente lo siguiente:

\section{Antartic Mineral. Resources}

The Representatives:

Recalling the provisions of the Antartic Treaty, which establishes a regime for international co-operation in Antarctica, with the objetive of ensuring that Antarctica should continue forever to be used exclusively for peaceful purposes and should not become the scene or object of international discord;

Bearing in mind the provisions of Article IV of the Treaty;

Convinced that the framework established by the Antarctic Treaty has proved effective in promoting international harmony in furtherance of the purposes and principles of the United Nations Charter, in ensuring the protection of the

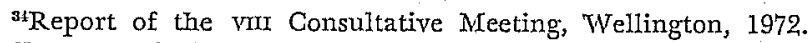

${ }^{35}$ Report of the IX Consultive Meeting, Oslo, 1975.

3o"Antarctic Resources", Report from the informal meeting of experts (30 May - 9 June, 1973), The Fridjof Nansen Foundation, Oslo:

TVer "Report of the Ninth Consultative Meeting", ya citado, página 56. 
Antarctic environment and on promoting freedom of scientific research in Antarctica;

Nothing with thanks the Report of the Scientific Committee on Antarctic Research (SCAR) Group of Specialists entitied Preliminary Assessment of the Environmentál Impact of Mineral Exploration Exploitation in Antarctica (EAMREA);

Recognizing nevertheless that adequate scientific data concerning the harmful environmental effects of activities related to the exploration and exploitation of Antarctic mineral resources, should they occur, are not yet available;

Concerned that unregulated activities related to exploration and exploitation of mineral resources could aciversely affect the unique environment of the Antarctic and other ecosystems dependent on the Antarctic environment;

Conscious that the Consultative Parties to the Antarctic Treaty in carrying out scientific research in the area have accumulated valuable experience and can substantially contribute to the protection of the environment and the rational use of Antarctic mineral resources, should exploration or exploitation there of occur;

Aware of the special responsibilities of Consultative Parties to ensure that any activities in Antarctica, including commexcial exploration and exploitation in the future, should they occur, should not become the cause of international discord, of danger to the unique Antarctic environment, of disruption to scientific investigation, or be otherwise contrary to the principles or purposes of the Antarictic Treaty;

Recommend to their Governments that:

1. They reaffirm the basic principles set forth in Recommendation vul-14 of the Eighth Antarctic Treaty Consultative Meeting;

2. They take note with appreciation of the Report of the Group of Experts on Mineral Exploration and Exploitation annexed to the Report of the Ninth Consultative Meeting and make the best possible use of its conclusions and guidelines;

3. They continue to study the environmental implications of mineral resources activities in the Antarctic Treaty Area and hold at a time and place to be arranged through diplomatic channels a meeting of ecological, technological and other related experts, in accordance with Recommendation $\mathrm{rV}-24$, with a view to developing scientific programmes aimed at:

(i) improving predictions of the impact of possible technologies for mineral exploration and exploitation in the Antarctic, as outlined in Section IIB of the Report of the Group of Experts and in Section 5 of the SCAR/EAMREA Group Report:

(ii) developing measures for the prevention of damage to the environment or for its rehabilitation, in accordance with. Section Irc of the Report of the Group of Experts;

4. They endorse the following principles elaborated at the Special Preparatory Meeting held in Paris from 28 June to 10 July 1976: 
(i) the Consultative Parties will continue to play in active and responsible role in dealing with the question of the mineral resources of Antarctica;

(ii) the Antarctic Treaty must be maintained in its entirety;

(iii) protection of the unique Antarctic environment and of its dependent ecosystems should be a basic consideration;

(iv) the Consultative Parties, in dealing with the question of mineral resources in Antarctica, should not prejudice the interets of all mankind in Antarctica;

5. They note that the provisions of Article $\mathrm{NV}$ of the Antarctic Treaty shall not be affected by the regime. It should ensure that the principles embodied in Article IV of the Antarctic Treaty are safeguarded in application to the area covered by the Antarctic Treaty;

6. They study the content of a future regime based on the principles contained in paragraphs 4 and 5 and on such further principles, rules and arrangements as may be agreed, taking full account of all propasals submitted to the Ixth Consultative Meeting;

7. The subject "Antarctic Resources - The Question of Mineral Exploration and Exploitation" be the subject of intensified consultation among them and they urge the host Government of the Tenth Consultative Meeting to convene a meeting to consider legal and political aspects of mineral resource issues; this meeting to report to the Tenth Consultative Meeting on the results of its work; 8. They urge their nationals and other States to refrain from all exploration and exploitation of Antarctic mineral resources while making progress towards the timely adoption of an agreed regime concerning Antarctic mineral resource activities. They will thus encleavour to ensure that, pending the timely adoption of agreed solutions pertaining to exploration and exploitation of mineral resources, no activity shall be conducted to explore or exploit such resources. They will keep the matters under continuing examination;

9. The subject "Antarctic Resources-The Question of Mineral Exploration and Exploitation" be placed on the Agenda of the Tenth Antarctic Treaty Consultative Meeting.

53. Los principios expresados en el punto 4) de la Recomendación que se ha reproducido tienen la mayor importancia $y$ deberán orientar la actividad futura de las Partes Consultivas.

Entre ellos se reitera el papel activo y responsable de las mismas Partes Consultivas, que deberán tratar la cuestión protegiendo al singular medio ambiente antártico y sus ecosistemas dependientes, sin perjudicar los intereses de toda la Humanidad. Agregan que los principios del Artículo Iv del Tratado Antártico - relativos a las reclamaciones de soberanía- deben ser salvaguardados en su aplicación al área cubierta por el Tratado Antártico. 
54. Las Partes Consultivas han acordado dedicar atención preferente en su Décima Reunión Consultiva, que deberá tener lugar en Washington en 1979, y sus Reuniones Preparatorias, a la cuestión de la exploración y explotación minerales en su relación con el medio ambiente.

\section{CONGLUSIONES}

55. Fluye de todo lo anterior que la Antártica no es un territorio ausente de normas jurídicas, remoto al hombre y "res nullius".

Por el contrario, hay un verdadero Sistema Antártico que se integra perfectamente al sistema internacional general, corresponde a los principios y propósitos de las Naciones Unidas y ha demos. trado su eficacia en el espacio y en el tiempo.

56. Por consiguiente, es por medio de dicho sistema antártico y en estrecha vinculación con él, que debe encontrarse la solución a la cuestión del aprovechamiento de los recursos del área.

Es necesario mantener y fortalecer el Tratado Antártico, a cuyo amparo se ha conocido, preservado y desarrollado una zona extrema del planeta. Resulta esencial que se preserve la reserva ecológica creada bajo el marco de ese instrumento y el laboratorio científico abierto en el área a todos los hombres. Tiene también una gran importancia el que la Antártica sea mantenida como una zona de paz, ajena a los vaivenes de las pugnas de poder y de las luchas militares; y que se perpetúe y extienda, si fuere posible, la cooperación entre Estados en la zona, cuyo carácter es único.

57. El Sistema Antártico se ha demostrado capaz de enfrentar con éxito, durante casi dos decenios, los problemas surgidos en la Antártica.

En el caso concreto de los recursos vivos y minerales $-\mathrm{y}$ su inseparable relación con los problemas del medio ambiente-, las Partes Consultivas han adoptado todos los pasos conducentes a un estudio y solución ađecuados.

En las páginas anteriores se ha descrito sumariamente lo obrado dentro del marco del Tratado Antártico, que aparece como una excelente vía de solución a la cuestión de aprovechamiento de los referidos recursos.

58. La solución debe originarse y ser propuesta por el Sistema Antártico. Es necesario que ella resulte -en cada caso- de un estudio profundizado y de una negociación suficientemente madura. 
59. Los posibles regímenes para el aprovechamiento de los recursos vivos y minerales deberán tener en cuenta el carácter singular del ecosistema antártico y su relación con otros ecosistemas, las realidades jurídicas y políticas del Ilamado Sistemá Antártico y el interés de la Humanidad. No podrán ignorar, junto a la realidad del Tratado Antártico y comprendida en él, la existencia de reclamaciones soberanas y la larga presencia antártica de muchos pafses, que han invertido en el conocimiento y desarrollo de este Continente el esfuerzo y el talento de sus hombres e ingentes recursos.

60. En el caso de los recursos vivos, la solución preparada por las Partes Consultivas estudiada anteriormente, parece ser el camino más racional y tal vez el único posible para la obtención de los objetivos señalados. Una Convención originada en el Sistema Antártico pero abierta a todos los Estados interesados, que ponga su acento en la conservación de los recursos vivos y en la preservación del ecosistema, aparece como un camino realista y justo.

61. En cuanto a los recursos minerales, es evidente que se requiere mucho más estudio y antecedentes respecto al posible efecto ecológico de su eventual exploración o explotación. - La continuación de la moratoria de hecho que existe a su respecto resulta esencial, tratándose de un medio ambiente tan delicado y con carácter tan singular.

Junto a ese estudio es preciso dar una solución apropiadai al delicado problema de los derechos de soberanía existentes o reclamados en la Antártica, en su aplicación a este caso concreto.

Todo ello supone una negociación difícil, que requerirá esfuerzos, imaginación, paciencia y flexibilidad de parte de todos los interesados.

El marco ideal -y probablemente el único adecuado- es, una vez más, el del Sistema Antártico, Los principios y parámetros adoptados en la Novena Reunión Consultiva de Londres, que han sido transcritos anteriormente, parecen ofrecer la base adecuada para este complejo ejercicio.

62. Toda otra solución -y especialmente una que fuese procura. da fuera del marco del Tratado Ántártico-, no podría ser efectiva $y$, si se intentase, podría acarrear graves consecuencias para todo lo obtenido en la Antártica en tantos años de labor.

Tampoco cabe, a nuestro juicio, el anticipar fórmulas completas. de carácter teórico. Es preciso que la solución resulte de la realidad antártica, de Ios estudios en curso y de las negociaciones que necesariamente deberán tener lugar. 
63. Al concluir estas observaciones generales, limitadas por la extensión y dificultades del tema a la vez que por el apremio del tiempo, deseo reiterar mis agradecimientos a la Revista de Derecho y la Universidad de Miami.

Quiero, también, reafirma que los comentarios precedentes tienen un carácter preliminar y estrictamente personal y han sido formulados con el solo propósito de aportar una contribución al interesante estudio iniciado bajo el patrocinio de la revista.

NuEva York, 14 de noviembre de 1978. 